\title{
Shimura correspondence of Maass wave forms of half integral weight
}

\author{
by \\ HisAshi KoJima (Tokyo)
}

\begin{abstract}
Introduction. In [11], Shimura showed the existence of a correspondence between modular forms of half integral weight and those of integral weight which commutes with the action of Hecke operators, and he exhibited some close relations between them. Using theta series attached to quadratic forms, Shintani [12] gave an inverse mapping to the Shimura mapping. Following the ideas of Shintani, Niwa [7] reformulated the Shimura correspondence by representing it as the inner product of modular forms of half integral weight. This Shintani-Niwa's theta lifting was generalized to the case of holomorphic modular forms on the tube domain associated with quadratic forms of signature $(2, n-2)$ by Oda [8] and by Rallis and Schiffmann [9]. These liftings only concern the case of holomorphic modular forms. On the other hand, Asai [1] and Friedberg [3] and [4] treated the theta lifting for non-holomorphic modular forms. Thus, a question arises whether we can generalize the Shimura correspondence to such forms.

The purpose of this note is to derive a correspondence $\Psi$ between nonholomorphic modular forms $F$ of half integral weight and those of integral weight and to describe an explicit relation between the Fourier coefficients of $F$ and $\Psi(F)$. Our results yield some generalizations of Shimura's result [11].

Section 1 is of preparatory character: we recall the definition of and basic results on Maass wave forms of integral weight and half integral weight. In Section 2, we summarize the definition and properties of the theta series $\Theta(z, g)$ introduced in [7] and [12]. Using $\Theta(z, g)$, we construct a mapping $\Psi$ of Maass wave forms of half integral weight to those of integral weight. In Section 3, we introduce some theta series and we derive a transformation formula for them. In that section we employ some methods of Niwa. We verify that $\Theta(z, g)$ splits into some simpler theta series. Niwa [7] showed this decomposition only for diagonal elements $g=\left(\begin{array}{l}* \\ 0\end{array}\right) \in \mathrm{SL}(2, \mathbb{R})$, which was sufficient for computation of the Fourier coefficients of the image of the Shimura lifting of holomorphic modular forms of half integral weight. We
\end{abstract}


need the decomposition of $\Theta(z, g)$ for all $g \in \mathrm{SL}(2, \mathbb{R})$. It plays an important role in our later discussion.

In Section 4, using the results of Section 3 on $\Theta(z, g)$, we determine the Fourier coefficients of $\Psi(F)$ in terms of those of $F$ for Maass wave forms $F$ of half integral weight. Moreover, we show that $\Psi$ commutes with the action of Hecke operators. In Section 5, we study the cuspidal properties of the image of $\Psi$.

We mention that Gelbert and Piatetski-Shapiro [5] and Waldspurger [13] generalized Shimura's results to the case of an arbitrary global field in terms of representation theory. Our results seem to be contained in their general theory. However, we emphasize that our correspondence can be described explicitly in terms of the Fourier coefficients of modular forms.

In [6], Hejhal obtained an analogous result to our Theorem 1 in a more general form. His lifting by means of theta series is a mapping from Maass wave forms with respect to Fuchsian groups with compact quotient space to those with respect to Fuchsian groups with non-compact quotient. So there is no overlap between Hejhal's results and ours.

Finally, the author is indebted to the referee for suggesting some revisions of this paper.

1. Notation and preliminaries. We denote by $\mathbb{Z}, \mathbb{Q}, \mathbb{R}$ and $\mathbb{C}$ the ring of rational integers, the rational number field, the real number field and the complex number field, respectively. For $z \in \mathbb{C}$, we put $e[z]=\exp (2 \pi i z)$ with $i=\sqrt{-1}$ and define $\sqrt{z}=z^{1 / 2}$ so that $-\pi / 2<\arg z^{1 / 2} \leq \pi / 2$. Moreover, we put $z^{\kappa / 2}=\left(z^{1 / 2}\right)^{\kappa}$ for every $\kappa \in \mathbb{Z}$.

This section is devoted to summarizing notation and several facts on nonholomorphic modular forms of integral weight and half integral weight (cf. Friedberg [4] and Shimura [11]). Let $\mathrm{GL}^{+}(2, \mathbb{R})($ resp. SL $(2, \mathbb{R}))$ be the group of all real matrices of degree 2 with positive determinant (resp. determinant one) and $\mathfrak{H}$ the complex upper half plane, i.e., $\mathfrak{H}=\{z \in \mathbb{C} \mid \operatorname{Im} z>0\}$. Define an action of $\mathrm{GL}^{+}(2, \mathbb{R})$ on $\mathfrak{H}$ by

$$
z \mapsto \sigma(z)=(a z+b)(c z+d)^{-1}
$$

for every $\sigma=\left(\begin{array}{ll}a & b \\ c & d\end{array}\right) \in \mathrm{GL}^{+}(2, \mathbb{R})$. For a positive integer $N$, put

$$
\begin{gathered}
\Gamma(N)=\left\{\sigma=\left(\begin{array}{ll}
a & b \\
c & d
\end{array}\right) \in \mathrm{SL}(2, \mathbb{R}) \mid a, b, c, d \in \mathbb{Z}\right. \text { and } \\
\left.\sigma \equiv\left(\begin{array}{ll}
1 & 0 \\
0 & 1
\end{array}\right)(\bmod N)\right\}, \\
\Gamma_{0}(N)=\left\{\sigma=\left(\begin{array}{ll}
a & b \\
c & d
\end{array}\right) \in \mathrm{SL}(2, \mathbb{R}) \mid a, b, c, d \in \mathbb{Z} \text { and } c \equiv 0(\bmod N)\right\}
\end{gathered}
$$


and $\operatorname{SL}(2, \mathbb{Z})=\Gamma(1)$. For a function $f$ on $\mathfrak{H}$ and a matrix $\sigma=\left(\begin{array}{ll}a & b \\ c & d\end{array}\right) \in$ $\mathrm{GL}^{+}(2, \mathbb{R})$ and for a half integer $k \in(1 / 2) \mathbb{Z}$, we define a function $f \mid[\sigma]_{k}$ on $\mathfrak{H}$ by

$$
f \mid[\sigma]_{k}(z)=(\operatorname{det} \sigma)^{k / 2}(c z+d)^{-k} f(\sigma(z)) .
$$

Now, consider a differential operator

$$
\Omega=\Omega^{k, \lambda}=-y^{2}\left(\frac{\partial^{2}}{\partial x^{2}}+\frac{\partial^{2}}{\partial y^{2}}\right)+k y i\left(\frac{\partial}{\partial x}+i \frac{\partial}{\partial y}\right)+\lambda
$$

with $z=x+i y \in \mathfrak{H}$, where $k \in(1 / 2) \mathbb{Z}$ and $\lambda \in \mathbb{C}$. The following property may be easily checked:

$$
\left(\Omega^{k, \lambda} f\right) \mid[\sigma]_{k}=\Omega^{k, \lambda}\left(f \mid[\sigma]_{k}\right)
$$

for every $\sigma \in \mathrm{GL}^{+}(2, \mathbb{R})$ and all twice differentiable functions $f$ on $\mathfrak{H}$.

Let $k$ be a positive integer. We call a real analytic function $f$ on $\mathfrak{H}$ a Maass wave form of weight $k$ with respect to $\Gamma(N)$ if:

$$
\begin{gathered}
\Omega^{k, \lambda} f=0, \\
f \mid[\sigma]_{k}=f \quad \text { for every } \sigma \in \Gamma(N),
\end{gathered}
$$

(1.6) for all $A \in \mathrm{SL}(2, \mathbb{Z})$, there is a $\delta>0$ such that

$$
f \mid[A]_{k}(z)=O\left(y^{\delta}\right)
$$

uniformly in $x$ as $y \rightarrow \infty$.

We denote by $G\{N, k, \lambda\}$ the space of all such forms $f$. The subspace $S\{N, k, \lambda\} \subset G\{N, k, \lambda\}$ consists of functions $f$ such that

(1.7) for all $A \in \mathrm{SL}(2, \mathbb{Z})$, there is a fixed $\delta>0$ with $f \mid[A]_{k}(z)=O\left(e^{-\delta y}\right)$ uniformly in $x$ as $y \rightarrow \infty$.

For a character $\chi$ modulo $N$, we also set

$$
\begin{aligned}
& G_{k, \lambda}(N, \chi)=\left\{f \in G\{N, k, \lambda\}|f|[\sigma]_{k}=\chi(d) f\right. \\
&\text { for every } \left.\sigma=\left(\begin{array}{ll}
a & b \\
c & d
\end{array}\right) \in \Gamma_{0}(N)\right\}
\end{aligned}
$$

and

$$
S_{k, \lambda}(N, \chi)=G_{k, \lambda}(N, \chi) \cap S\{N, k, \lambda\} .
$$

Let $W_{m, n}$ be a Whittaker function, a solution of a differential equation

$$
\left(4 y^{2} \frac{\partial^{2}}{\partial y^{2}}+\left(1-4 n^{2}\right)+4 m y-y^{2}\right) W_{m, n}(y)=0,
$$

which goes to $y^{m} e^{-y / 2}$ as $y \rightarrow \infty$, where $m \in \mathbb{R}$ and $n \in \mathbb{C}$. For $\varepsilon= \pm 1$, 
$y>0, k \in \mathbb{R}$ and for $\lambda \in \mathbb{C}$, set

$$
\begin{aligned}
\omega & =\left((k-1)^{2}+4 \lambda\right)^{1 / 2} / 2, \\
W(\varepsilon y, k, \lambda) & =y^{-k / 2} W_{\varepsilon k / 2, \omega}(2 y), \\
u(y, k, \lambda) & = \begin{cases}y^{((1-k) / 2-\omega)} & \text { if } \omega \neq 0, \\
y^{(1-k) / 2} \log y & \text { otherwise. }\end{cases}
\end{aligned}
$$

The following lemma is given in [4].

Lemma 1.1. Let $F \in G\{N, k, \lambda\}$ and $F^{\prime} \in G_{k, \lambda}(N, \chi)$. Then:

(i) $F$ has the following Fourier expansion at the cusp corresponding to $A \in \mathrm{SL}(2, \mathbb{Z})$ :

$$
\begin{aligned}
F \mid\left[A^{-1}\right]_{k}(z)= & a_{A}(0) y^{((1-k) / 2+\omega)}+a_{A}^{\prime}(0) u(y, k, \lambda) \\
& +\sum_{n \neq 0} a_{A}(n) W(2 \pi n y / N, k, \lambda) e[n x / N],
\end{aligned}
$$

where $\sum_{n \neq 0}$ is the sum taken over all $n \in \mathbb{Z}-\{0\}$,

(ii) $F^{\prime}$ has the following Fourier expansion at infinity:

$$
\begin{aligned}
F^{\prime}(z)= & a(0) y^{((1-k) / 2+\omega)}+a^{\prime}(0) u(y, k, \lambda) \\
& +\sum_{n \neq 0} a(n) W(2 \pi n y, k, \lambda) e[n x] .
\end{aligned}
$$

For $k \in \mathbb{Z}$ and $\lambda \in \mathbb{C}$, put

$M\{N, k, \lambda\}=\left\{f: \mathfrak{H} \rightarrow \mathbb{C} \mid f\right.$ is real analytic, $\Omega^{k, \lambda} f=0$ and

$$
\left.f \mid[\sigma]_{k}=f \text { for every } \sigma \in \Gamma(N)\right\} .
$$

Then we can easily check the following lemma.

Lemma 1.2. Suppose that $f \in M\{N, k, \lambda\}$ satisfies:

(i) for some fixed $\nu>0,|f(z)|=O\left(y^{-\nu}\right)$ uniformly in $x$ as $y \rightarrow \infty$,

(ii) for some fixed $\nu^{\prime}>0,|f(z)|=O\left(y^{-\nu^{\prime}}\right)$ uniformly in $x$ as $y \rightarrow 0$.

Then $f \in G\{N, k, \lambda\}$.

We may derive the following lemma.

Lemma 1.3. Let $f \in G\{N, k, \lambda\}$ and $A \in \mathrm{SL}(2, \mathbb{Z})$. Suppose that, for some $\nu^{\prime}>0, f \mid\left[A^{-1}\right]_{k}(z)=O\left(y^{-\nu^{\prime}}\right)$ uniformly in $x(|x| \leq N / 2)$ as $y \rightarrow 0$. Then there is a positive number $\alpha$ such that $a_{A}(n)=O\left(|n|^{\alpha}\right)$.

Pr o o f. By Lemma 1.1, $f \mid\left[A^{-1}\right]_{k}$ has the Fourier expansion

$$
f \mid\left[A^{-1}\right]_{k}(z)=a_{0}(y)+\sum_{n \neq 0} a_{A}(n) W(2 \pi n y / N, k, \lambda) e[n x / N] .
$$


For $n \neq 0$, we have

$$
a_{A}(n)=(1 / N) \int_{-N / 2}^{N / 2} f \mid\left[A^{-1}\right]_{k}(z) W(2 \pi n y / N, k, \lambda)^{-1} e[-n x / N] d x .
$$

Combining this with the assumption above, we have

$$
\left|a_{A}(n)\right| \leq M y^{-\nu^{\prime}}|W(2 \pi n y / N, k, \lambda)|^{-1}
$$

for some $M$. Put $y=N /|n|$ with $|n|$ sufficiently large. Then

$$
\left|a_{A}(n)\right| \leq M(N /|n|)^{-\nu^{\prime}}(2 \pi)^{-k / 2}\left|W_{\operatorname{sgn}(n)(k / 2), \omega}(2 \pi)\right|^{-1} .
$$

Since

$W_{m, n}(z)=z^{m} e^{-z / 2}(\Gamma(1 / 2-m+n))^{-1} \int_{0}^{\infty} t^{-m-1 / 2+n}(1+t / z)^{m-1 / 2+n} e^{-t} d t$ for every $z>0$, we get the assertion of the lemma.

By the same argument as in the case of holomorphic modular forms, we can prove the following lemma (cf. [2]).

LEMma 1.4. If $f \in S\{N, k, \lambda\}$, then there is a constant $M$ such that

$$
|f(z)| \leq M y^{-k / 2} \quad \text { for every } z \in \mathfrak{H} .
$$

Define a Hecke operator $T_{k, \lambda, \chi}^{N}(n)$ on $G_{k, \lambda}(N, \chi)$ by

$$
f \mid T_{k, \lambda, \chi}^{N}(n)(z)=n^{k-1} \sum_{d} \sum_{b=0}^{d-1} \chi((n / d)) d^{-k} f\left(\frac{(n / d) z+b}{d}\right),
$$

where $n$ is a positive integer and $\sum_{d}$ is the sum taken over all positive divisors $d$ of $n$. Then the following may be easily checked (cf. [2]).

Lemma 1.5. Let $f \in G_{k, \lambda}(N, \chi)$. Suppose that $f(z)$ and $f \mid T_{k, \lambda, \chi}^{N}(m)(z)$ have the Fourier expansions

$$
f(z)=\sum_{n \neq 0} a(n) W(2 \pi n y, k, \lambda) e[n x]
$$

and

$$
f \mid T_{k, \lambda, \chi}^{N}(m)(z)=\sum_{n \neq 0} b(n) W(2 \pi n y, k, \lambda) e[n x],
$$

where $\sum_{n \neq 0}$ is the sum taken over all $n \in \mathbb{Z}-\{0\}$. Then

$$
b(n)=\sum_{d} \chi(d) d^{k-1} a\left(m n / d^{2}\right),
$$

where the summation is taken over all positive divisors $d$ of $m$ and $n$.

The remainder of this section is devoted to explaining the notion of nonholomorphic modular forms of half integral weight (cf. [11]). Let $G$ denote 
the set of all couples $(\alpha, \varphi(z))$ formed by an element $\alpha=\left(\begin{array}{ll}a & b \\ c & d\end{array}\right)$ of $\mathrm{GL}^{+}(2, \mathbb{R})$ and a holomorphic function $\varphi(z)$ on $\mathfrak{H}$ such that

$$
\varphi(z)^{2}=t(\operatorname{det} \alpha)^{-1 / 2}(c z+d)
$$

with $t \in T$, where $T=\{z \in \mathbb{C}|| z \mid=1\}$. We make $G$ a group by defining

$$
(\alpha, \varphi(z)) \cdot(\beta, \psi(z))=(\alpha \beta, \varphi(\beta(z)) \cdot \psi(z)) .
$$

For $\sigma=\left(\begin{array}{ll}a & b \\ c & d\end{array}\right) \in \Gamma_{0}(4)$ and $z \in \mathfrak{H}$, we define an automorphic factor $J(\sigma, z)$ by

$$
J(\sigma, z)=\varepsilon_{d}^{-1}\left(\frac{c}{d}\right)(c z+d)^{1 / 2},
$$

where

$$
\varepsilon_{d}= \begin{cases}1 & \text { if } d \equiv 1(\bmod 4) \\ i & \text { otherwise }\end{cases}
$$

and $\left(\frac{c}{d}\right)$ is the symbol given in [11]. Let $\kappa=2 \lambda+1$ be an odd integer and $\nu$ a complex number. Moreover, let $\psi$ be a character modulo $4 N$. We say that a function $F$ on $\mathfrak{H}$ is a Maass wave form of Neben-type $\psi$ and of half integral weight with respect to $\Gamma_{0}(4 N)$ if the following conditions are satisfied:

$$
F \text { is real analytic, }
$$

$$
\begin{gathered}
F(\sigma(z))=\psi(d) J(\sigma, z)^{\kappa} F(z) \quad \text { for every } \sigma=\left(\begin{array}{ll}
a & b \\
c & d
\end{array}\right) \in \Gamma_{0}(4 N), \\
\Omega^{\kappa / 2, \nu} F=0,
\end{gathered}
$$

(1.13) for $A=\left(\begin{array}{ll}a & b \\ c & d\end{array}\right) \in \mathrm{SL}(2, \mathbb{Z})$ and for some $\delta>0$,

$$
F(A(z))(c z+d)^{\kappa / 2}=O\left(e^{-\delta y}\right) \quad \text { uniformly in } x \text { as } y \rightarrow \infty .
$$

We denote by $S_{\kappa / 2, \nu}(4 N, \psi)$ the space of all such $F$. From the definition, $F \in S_{\kappa / 2, \nu}(4 N, \psi)$ has the Fourier expansion

$$
F(z)=\sum_{n \neq 0} a(n) W(2 \pi n y, \kappa / 2, \nu) e[n x] .
$$

Let $m$ be the square of a positive integer and let

$$
\alpha=\left(\begin{array}{cc}
1 & 0 \\
0 & m
\end{array}\right), \quad \xi=\left(\alpha, m^{1 / 4}\right) .
$$

Put $\Gamma_{0}(4 N)=\left\{(\sigma, J(\sigma, z)) \mid \sigma \in \Gamma_{0}(4 N)\right\}$. Then $\Delta_{0}(4 N) \xi \Delta_{0}(4 N)$ can be expressed as a finite disjoint union:

$$
\Delta_{0}(4 N) \xi \Delta_{0}(4 N)=\bigcup_{\nu=1}^{r} \Delta_{0}(4 N) \xi_{\nu} .
$$


We define a Hecke operator $T_{\kappa / 2, \nu, \psi}^{4 N}(m)$ on $S_{\kappa / 2, \nu}(4 N, \psi)$ by

$$
F \mid T_{\kappa / 2, \nu, \psi}^{4 N}(m)(z)=m^{\kappa / 4-1} \sum_{\nu=1}^{r} \psi\left(a_{\nu}\right) F\left(\alpha_{\nu}(z)\right)\left(\psi_{\nu}(z)\right)^{-\kappa}
$$

for every $F \in S_{\kappa / 2, \nu}(4 N, \psi)$, where $\xi_{\nu}=\left(\alpha_{\nu}, \psi_{\nu}(z)\right)$. A slight modification of arguments in [11] yields the following (cf. [11, Theorem 1.7 and Corollary 1.7]).

Lemma 1.6. Let $p$ be a prime number and $F \in S_{\kappa / 2, \nu}(4 N, \psi)$. Set

$$
F(z)=\sum_{n \neq 0} a(n) W(2 \pi n y, \kappa / 2, \nu) e[n x]
$$

and

$$
F \mid T_{\kappa / 2, \nu, \psi}^{4 N}\left(p^{2}\right)(z)=\sum_{n \neq 0} b(n) W(2 \pi n y, \kappa / 2, \nu) e[n x] .
$$

Then

(1.14) $b(n)=a\left(p^{2} n\right)+\psi(p)\left(\frac{-1}{p}\right)^{(\kappa-1) / 2} p^{(\kappa-3) / 2} a(n)+\psi\left(p^{2}\right) p^{\kappa-2} a\left(n / p^{2}\right)$,

where $a\left(n / p^{2}\right)$ means 0 if $p^{2}$ does not divide $n$.

2. Theta series constructed from the Weil representation. To define the Shimura correspondence of Maass wave forms, we consider theta series introduced by Shintani [12]. Let $N$ be a positive integer and $\chi$ a character modulo $4 N$, and let $\lambda$ be a positive integer. Put $\chi_{1}=\chi\left(\frac{-1}{*}\right)^{\lambda}$ and $\kappa=2 \lambda+1$. Define a representation $\varrho$ of $\operatorname{SL}(2, \mathbb{R})$ on $\mathbb{R}^{3}$ by

$$
\varrho(g) \cdot x=\left(x_{1}, x_{2}, x_{3}\right)\left(\begin{array}{ccc}
a^{2} & 2 a c & c^{2} \\
a b & a d+b c & c d \\
b^{2} & 2 b d & d^{2}
\end{array}\right)
$$

for every $g=\left(\begin{array}{ll}a & b \\ c & d\end{array}\right) \in \mathrm{SL}(2, \mathbb{R})$ and $x=\left(x_{1}, x_{2}, x_{3}\right) \in \mathbb{R}^{3}$. For $x \in \mathbb{R}^{3}$, we put

$$
f(x)=\left(x_{1}-i x_{2}-x_{3}\right)^{\lambda} \exp \left((-2 \pi / N)\left(2 x_{1}^{2}+x_{2}^{2}+2 x_{3}^{2}\right)\right) .
$$

Set

$$
L=\left\{x=\left(x_{1}, x_{2}, x_{3}\right) \in \mathbb{R}^{3} \mid x_{1} \in \mathbb{Z}, x_{2} \in N \mathbb{Z}, x_{3} \in(N / 4) \mathbb{Z}\right\} .
$$

Define a theta series $\Theta(z, g)$ on $\mathfrak{H} \times \mathrm{SL}(2, \mathbb{R})$ by

$$
\begin{aligned}
\Theta(z, g)= & \sum_{x \in L} \bar{\chi}_{1}\left(x_{1}\right) v^{(3-\kappa) / 4} \exp \left(2 \pi i(u / N)\left(x_{2}^{2}-4 x_{1} x_{3}\right)\right) \\
& \times f\left(\sqrt{v}\left(\varrho\left(g^{-1}\right) \cdot x\right)\right)
\end{aligned}
$$

with $g \in \mathrm{SL}(2, \mathbb{R})$ and $z=u+i v \in \mathfrak{H}$, where $x=\left(x_{1}, x_{2}, x_{3}\right) \in L$. 
Put

$$
w=\xi+i \eta \in \mathfrak{H} \quad \text { and } \quad \sigma_{w}=\left(\begin{array}{cc}
\sqrt{\eta} & \xi \sqrt{\eta}^{-1} \\
0 & \sqrt{\eta}^{-1}
\end{array}\right) \in \operatorname{SL}(2, \mathbb{R}) .
$$

Define a function $\Psi(F)$ on $\mathfrak{H}$ by

$$
\Psi(F)(w)=(4 \eta)^{-\lambda} \int_{\Gamma_{0}(4 N) \backslash \mathfrak{H}} v^{\kappa / 2} \bar{\Theta}\left(z, \sigma_{4 w}\right) F(z) v^{-2} d u d v
$$

for every $F \in S_{\kappa / 2, \nu}\left(4 N, \bar{\chi}\left(\frac{N}{*}\right)\right)$, where $z=u+i v \in \mathfrak{H}$.

The following proposition is shown in [10, pp. 303-304] (cf. [7]).

Proposition 2.1. Suppose $\kappa \geq 7$. Then $\Psi(F) \in G_{2 \lambda, 4 \nu}\left(2 N, \bar{\chi}^{2}\right)$.

The mapping

$$
S_{\kappa / 2, \nu}\left(4 N, \bar{\chi}\left(\frac{N}{*}\right)\right) \ni F \rightarrow \Psi(F) \in G_{2 \lambda, 4 \nu}\left(2 N, \bar{\chi}^{2}\right)
$$

is called the Shimura correspondence of Maass wave forms of half integral weight.

3. The decomposition of the theta series $\Theta(z, g)$. In this section, we try to express $\Theta(z, g)$ in another way. In [7], Niwa verified that $\Theta(z, g)$ splits into simpler theta series for a diagonal element $g=\left(\begin{array}{l}* \\ 0\end{array}\right)$. For our arguments we need to express $\Theta(z, g)$ in a convenient form for all $g \in \mathrm{SL}(2, \mathbb{R})$. By $[7$, p. 153], it is sufficient to consider only the elements of the form

$$
g=\left(\begin{array}{cc}
\sqrt{y} & x \sqrt{y} \\
0 & \sqrt{y}^{-1}
\end{array}\right) .
$$

In the remainder of this section, we assume that $g$ has the above form.

Now we recall the Hermite polynomial $H_{\varepsilon}(x)$ which plays a useful role in our arguments. For $0 \leq \varepsilon \in \mathbb{Z}$, we define

$$
H_{\varepsilon}(x)=(-1)^{\varepsilon} \exp \left(x^{2} / 2\right) \frac{d^{\varepsilon}}{d x^{\varepsilon}} \exp \left(-x^{2} / 2\right) .
$$

Observing that

$$
(a-i b)^{\lambda}=\sum_{\varepsilon=0}^{\lambda}\left(\begin{array}{l}
\lambda \\
\varepsilon
\end{array}\right) H_{\lambda-\varepsilon}(a) H_{\varepsilon}(b)(-i)^{\varepsilon},
$$

we have

$$
\exp \left(2 \pi i(u / N)\left(x_{2}^{\prime 2}-4 x_{1}^{\prime} x_{3}^{\prime}\right)\right) f\left(\sqrt{v}\left(\varrho\left(g^{-1}\right) \cdot x^{\prime}\right)\right)
$$




$$
\begin{aligned}
= & (\sqrt{N} /(2 \sqrt{2 \pi}))^{\lambda} \sum_{\varepsilon=0}^{\lambda}\left(\begin{array}{l}
\lambda \\
\varepsilon
\end{array}\right)(-i)^{\varepsilon} H_{\varepsilon}\left(\sqrt{2 \pi v}\left(x_{2}^{\prime}-2 x x_{3}^{\prime}\right)\right) \\
& \times \exp \left((\pi i / 2)\left(x_{2}^{\prime}-2 x x_{3}^{\prime}\right) z\right) H_{\lambda-\varepsilon}\left(\sqrt{2 \pi v}\left(\left(x_{1}^{\prime}-x x_{2}^{\prime}+x^{2} x_{3}^{\prime}\right) y^{-1}-y x_{3}^{\prime}\right)\right) \\
& \times \exp \left(-2 \pi i x_{3}^{\prime}\left(x_{1}^{\prime}-x x_{2}^{\prime}+x^{2} x_{3}^{\prime}\right) u\right) \\
& \times \exp \left(-\pi v\left(\left(\left(x_{1}^{\prime}-x x_{2}^{\prime}+x^{2} x_{3}^{\prime}\right) / y\right)^{2}+\left(y x_{3}^{\prime}\right)^{2}\right)\right),
\end{aligned}
$$

where $f(*)$ is the function in $(2.1), x^{\prime}=\left(x_{1}^{\prime}, x_{2}^{\prime}, x_{3}^{\prime}\right) \in \mathbb{R}^{3}, z=u+i v \in \mathfrak{H}$ and $g=\left(\begin{array}{c}\sqrt{y} x \sqrt{y}^{-1} \\ 0 \sqrt{y}^{-1}\end{array}\right) \in \operatorname{SL}(2, \mathbb{R})$. For $\varepsilon \in \mathbb{Z}(\varepsilon \geq 0), x_{3}^{\prime} \in(\sqrt{N} / 2) \mathbb{Z}$ and $m \in \mathbb{Z}$, we define a function $\Theta_{1, \varepsilon}\left(z, g,(-m x) /(8 N), x x_{3}^{\prime} / \sqrt{N}\right)$ by

$$
\begin{aligned}
& \Theta_{1, \varepsilon}\left(z, g,(-m x) /(8 N), x x_{3}^{\prime} / \sqrt{N}\right) \\
& =v^{-\varepsilon / 2} \sum_{x_{2}^{\prime} \in 2 \sqrt{N \mathbb{Z}}} H_{\varepsilon}\left(\sqrt{2 \pi v}\left(x_{2}^{\prime}-2 x x_{3}^{\prime}\right)\right) \\
& \quad \times \exp \left((\pi i z / 2)\left(x_{2}^{\prime}-2 x x_{3}^{\prime}\right)^{2}+2 \pi i m\left(-x x_{2}^{\prime}+x^{2} x_{3}^{\prime}\right) /(8 \sqrt{N})\right) .
\end{aligned}
$$

To derive the transformation formula for $\Theta_{1, \varepsilon}$, we introduce the following differential operator. For $\lambda \in \mathbb{R}$, we put

$$
\frac{\partial}{\partial z}=\frac{1}{2}\left(\frac{\partial}{\partial u}-i \frac{\partial}{\partial v}\right) \quad \text { and } \quad \delta_{\lambda}=\frac{1}{2 \pi i}\left(\frac{\lambda}{2 i v}+\frac{\partial}{\partial z}\right)
$$

with $z=u+i v \in \mathbb{C}$ and we also put

$$
\delta_{\lambda}^{l}=\delta_{\lambda+2(l-1)} \circ \ldots \circ \delta_{\lambda+2} \circ \delta_{\lambda} \quad \text { for } 0 \leq l \in \mathbb{Z} .
$$

The differential operator $\delta_{\lambda}^{l}$ satisfies

$$
\delta_{\lambda}^{l}\left(f \mid[\sigma]_{\lambda}\right)=\left(\delta_{\lambda}^{l} f\right) \mid[\sigma]_{\lambda+2 l}
$$

for every $\sigma \in \mathrm{GL}^{+}(2, \mathbb{R})$ and for every function $f$ on $\mathfrak{H}$, where $\lambda \in(1 / 2) \mathbb{Z}$.

Now we can prove the following lemma.

Lemma 3.1. Let $\sigma=\left(\begin{array}{ll}a & b \\ c & d\end{array}\right)$ be an element of $\Gamma_{0}(4 N)$. Then

$$
\begin{gathered}
\Theta_{1, \varepsilon}\left(\sigma(z), g,(-m x /(8 N)) a+\left(x x_{3}^{\prime} / \sqrt{N}\right) b,(-m x /(8 N)) c+\left(x x_{3}^{\prime} / \sqrt{N}\right) d\right) \\
=\left(\frac{N}{d}\right) J(\sigma, z)(c z+d)^{\varepsilon} \Theta_{1, \varepsilon}\left(z, g,(-m x) /(8 N), x x_{3}^{\prime} / \sqrt{N}\right) .
\end{gathered}
$$

Proof. We need the notation of [3, pp. 3 and 4]. For $Q, R$ and $L$ as in $[3$, pp. 3 and 4], take $Q=2 N, R=2 N$ and $L=Z$. Then

$$
\begin{aligned}
& \Theta_{1, \varepsilon}\left(z, g,(-m x) /(8 N), x x_{3}^{\prime} / \sqrt{N}\right) \\
& \quad=\Theta\left(z,(-m x) /(8 N), x x_{3}^{\prime} / \sqrt{N}, 1, \varepsilon\right) \begin{cases}1 & \text { if } \varepsilon=0, \\
\sqrt{2 \pi / N} & \text { if } \varepsilon=1,\end{cases}
\end{aligned}
$$


where $\Theta(z, *, *, *, *)$ is the same symbol as $\Theta(z, u, v, w, f)$ of [3, p. 4]. By using [3, Theorem 1.2], we prove our assertion for $\varepsilon=0$ and 1. Using the equality

$$
\begin{aligned}
\delta_{\varepsilon+1 / 2}\left(v^{-\varepsilon / 2} H_{\varepsilon}(\sqrt{2 \pi v} x)\right. & \left.\exp \left(\pi i z x^{2} / 2\right)\right) \\
& =\frac{1}{8 \pi} v^{-(\varepsilon+2) / 2} H_{\varepsilon+2}(\sqrt{2 \pi v} x) \exp \left(\pi i z x^{2} / 2\right)
\end{aligned}
$$

and combining this with (3.3), we get our assertion for all $\varepsilon$.

By definition, we see easily

$$
\begin{aligned}
\Theta_{1, \varepsilon}(z, g,(m x) /(8 N), & \left.\left(-x x_{3}^{\prime}\right) / \sqrt{N}\right) \\
& =(-1)^{\varepsilon} \Theta_{1, \varepsilon}\left(z, g,(-m x) /(8 N), x x_{3}^{\prime} / \sqrt{N}\right) .
\end{aligned}
$$

For $0 \leq \varepsilon \in \mathbb{Z}, m \in \mathbb{R}$ and $n \in \mathbb{R}$, we put

$t_{\varepsilon}(z, m, n)$

$$
=v^{1 / 2}(8 \pi v)^{-\varepsilon / 2} H_{\varepsilon}(\sqrt{2 \pi v}(m-n)) \exp \left(\pi i\left(u(-2 m n)+i v\left(m^{2}+n^{2}\right)\right)\right)
$$

with $z=u+i v \in \mathbb{C}$. The following formula is shown in [1, Lemma 9]:

$$
\delta_{\varepsilon}^{l} t_{\varepsilon}=t_{\varepsilon+2 l} \quad \text { for every } \varepsilon \in \mathbb{Z}(\varepsilon \geq 0) .
$$

We put

$\widetilde{L}=(2 / \sqrt{N}) L \quad$ and $\quad \widetilde{L}_{1}=\left\{\left(x_{2}^{\prime}, x_{3}^{\prime}\right) \mid x_{2}^{\prime} \in 2 \sqrt{N} \mathbb{Z}\right.$ and $\left.x_{3}^{\prime} \in(\sqrt{N} / 2) \mathbb{Z}\right\}$.

For $\left(x_{2}^{\prime}, x_{3}^{\prime}\right) \in \widetilde{L}_{1}$, we define a theta series $\Theta_{2, \varepsilon}^{\bar{\chi}}\left(z, g, x_{2}^{\prime}, x_{3}^{\prime}\right)$ by

$$
\Theta_{2, \varepsilon}^{\bar{\chi}}\left(z, g, x_{2}^{\prime}, x_{3}^{\prime}\right)=\sum_{x_{1}^{\prime} \in(2 / \sqrt{N}) \mathbb{Z}} \bar{\chi}_{1}\left((\sqrt{N} / 2) x_{1}^{\prime}\right) t_{\varepsilon}\left(z,\left(x_{1}^{\prime}-x x_{2}^{\prime}+x^{2} x_{3}^{\prime}\right) / y, y x_{3}^{\prime}\right) \text {. }
$$

Now we prove the following lemma.

Lemma 3.2. Suppose that $\bar{\chi}_{1}$ is a primitive character. Then

$$
\begin{aligned}
\sqrt{8 \pi v}{ }^{\varepsilon} \Theta_{2, \varepsilon}^{\bar{\chi}}\left(z, g, x_{2}^{\prime}, x_{3}^{\prime}\right) \\
=G\left(\bar{\chi}_{1}\right)(1 /(8 \sqrt{N})) i^{\varepsilon} y^{\varepsilon+1}\left(2 \pi v^{-1}\right)^{\varepsilon / 2} \sum_{m=-\infty}^{\infty} \chi_{1}(m)\left(x_{3}^{\prime} z+(m /(8 \sqrt{N}))^{\varepsilon}\right. \\
\quad \times \exp \left(-\pi y^{2} v^{-1}\left|x_{3}^{\prime} z+(m /(8 \sqrt{N}))\right|^{2}+2 \pi i\left(\left(-x x_{2}^{\prime}+x^{2} x_{3}^{\prime}\right) /(8 \sqrt{N})\right)\right),
\end{aligned}
$$

where $G\left(\bar{\chi}_{1}\right)$ is the Gauss sum of $\bar{\chi}_{1}$.

$\operatorname{Proof}$. For $\varepsilon=0$, the lemma follows from the Poisson summation formula. We have

$$
\Theta_{2,0}^{\bar{\chi}}\left(z, g, x_{2}^{\prime}, x_{3}^{\prime}\right)=\sum_{\alpha \in \mathbb{Z} / 4 N \mathbb{Z}} \bar{\chi}_{1}(\alpha) f_{\alpha}\left(z, g, x_{2}^{\prime}, x_{3}^{\prime}\right),
$$


where

$$
\begin{aligned}
f_{\alpha}\left(z, g, x_{2}^{\prime}, x_{3}^{\prime}\right)= & \sum_{m=-\infty}^{\infty} \exp \left(-2 \pi i u x_{3}^{\prime}\left((2 / \sqrt{N})(4 N m+\alpha)-x x_{2}^{\prime}+x^{2} x_{3}^{\prime}\right)\right. \\
& \left.-\pi v\left(\left((2 / \sqrt{N})(4 N m+\alpha)-x x_{2}^{\prime}+x^{2} x_{3}^{\prime}\right)^{2} y^{-2}+\left(y x_{3}^{\prime}\right)^{2}\right)\right) .
\end{aligned}
$$

Applying the Poisson summation formula for $f_{\alpha}\left(z, g, x_{2}^{\prime}, x_{3}^{\prime}\right)$, we obtain

$$
\begin{aligned}
\Theta_{2,0}^{\bar{\chi}}\left(z, g, x_{2}^{\prime}, x_{3}^{\prime}\right)= & \sqrt{v}(1 /(8 \sqrt{N})) y \sqrt{v}^{-1} \sum_{\alpha \in \mathbb{Z} / 4 N \mathbb{Z}} \bar{\chi}_{1}(\alpha) \\
& \times \sum_{m=-\infty}^{\infty} \exp \left(-\pi y^{2} v^{-1}\left|x_{3}^{\prime} z+(m /(8 \sqrt{N}))\right|^{2}\right. \\
& \left.+2 \pi i m\left((2 / \sqrt{N}) \alpha-x x_{2}^{\prime}+x^{2} x_{3}^{\prime}\right) /(8 \sqrt{N})\right) .
\end{aligned}
$$

Since $\chi_{1}$ is primitive, we have

$$
\sum_{\alpha \in \mathbb{Z} / 4 N \mathbb{Z}} \bar{\chi}_{1}(\alpha) \exp (2 \pi i m \alpha /(4 N))=G\left(\bar{\chi}_{1}\right) \chi_{1}(m) .
$$

Consequently, we get our assertion in the case where $\varepsilon=0$. Operating with $\delta_{0}^{\varepsilon / 2}$ on the result above, we obtain the desired result for $\varepsilon$ even. For $\varepsilon=1$, adapting $\left(\sqrt{2 \pi} i x_{3}^{\prime} y\right)^{-1} \sqrt{v} \frac{\partial}{\partial u}$ to the case $\varepsilon=0$ and adding $x_{3}^{\prime} y(2 \pi v)^{1 / 2}$ times the result for $\varepsilon=0$, we obtain the assertion. For $\varepsilon$ odd, it is sufficient to operate with $\delta_{1}^{(\varepsilon-1) / 2}$ on the results above. This completes the proof.

The formula (3.1) and Lemma 3.2 imply

$$
\begin{aligned}
\Theta(z, g)= & (\sqrt{N} /(2 \sqrt{2 \pi}))^{\lambda} v^{-\lambda / 2} \sum_{\varepsilon=0}^{\lambda}\left(\begin{array}{l}
\lambda \\
\varepsilon
\end{array}\right)(-i)^{\varepsilon} \\
& \times \sum_{\left(x_{2}^{\prime}, x_{3}^{\prime}\right) \in \widetilde{L}_{1}} H_{\varepsilon}\left(\sqrt{2 \pi v}\left(x_{2}^{\prime}-2 x x_{3}^{\prime}\right)\right) \\
& \times \exp \left(\pi i\left(x_{2}^{\prime}-2 x x_{3}^{\prime}\right)^{2} z / 2\right) v^{1 / 2} \sum_{x_{1}^{\prime} \in(2 / \sqrt{N}) \mathbb{Z}} \bar{\chi}_{1}\left((\sqrt{N} / 2) x_{1}^{\prime}\right) \\
& \times H_{\lambda-\varepsilon}\left(\sqrt{2 \pi v}\left(\left(x_{1}^{\prime}-x x_{2}^{\prime}+x^{2} x_{3}^{\prime}\right) y^{-1}-y x_{3}^{\prime}\right)\right) \\
& \times \exp \left(-2 \pi i x_{3}^{\prime}\left(x_{1}^{\prime}-x x_{2}^{\prime}+x^{2} x_{3}^{\prime}\right) u\right) \\
& \times \exp \left(-\pi v\left(\left(\left(x_{1}^{\prime}-x x_{2}^{\prime}+x^{2} x_{3}^{\prime}\right) / y\right)^{2}+\left(y x_{3}^{\prime}\right)^{2}\right)\right) \\
= & (\sqrt{N} /(2 \sqrt{2 \pi}))^{\lambda} v^{-\lambda / 2} G\left(\bar{\chi}_{1}\right)(1 /(8 \sqrt{N})) \\
& \times \sum_{\varepsilon=0}^{\lambda}\left(\begin{array}{l}
\lambda \\
\varepsilon
\end{array}\right)(-i)^{\varepsilon} i^{\lambda-\varepsilon} y^{\lambda-\varepsilon+1} \varphi_{\varepsilon}(z, g)
\end{aligned}
$$


where

$$
\begin{aligned}
\varphi_{\varepsilon}(z, g)= & \left(2 \pi v^{-1}\right)^{(\lambda-\varepsilon) / 2} v^{\varepsilon / 2} \\
& \times \sum_{m \in \mathbb{Z}} \sum_{x_{3}^{\prime} \in(\sqrt{N} / 2) \mathbb{Z}} \Theta_{1, \varepsilon}\left(z, g,(-m x) /(8 N), x x_{3}^{\prime} / \sqrt{N}\right) \\
& \times \chi_{1}(m)\left(x_{3}^{\prime} \bar{z}+(m /(8 \sqrt{N}))\right)^{\lambda-\varepsilon} \\
& \times \exp \left(-\pi y^{2} v^{-1}\left|x_{3}^{\prime} \bar{z}+(m /(8 \sqrt{N}))\right|^{2}\right) .
\end{aligned}
$$

Let $W_{4 N}$ be the set of all couples $\left(4 N x_{3}^{\prime}, m\right)$ of integers such that either $m>0, x_{3}^{\prime} \in \mathbb{Z}$ and $\left(4 N x_{3}^{\prime}, m\right)=1$, or $m=x_{3}^{\prime}=0$. Then the mapping $\Gamma_{\infty}\left(\begin{array}{ll}a & b \\ c & d\end{array}\right) \mapsto(c, d)$ gives an isomorphic mapping between $\Gamma_{\infty} \backslash \Gamma_{0}(4 N)$ and $W_{4 N}$, where $\Gamma_{\infty}=\left\{ \pm\left(\begin{array}{ll}1 & n \\ 0 & 1\end{array}\right) \mid n \in \mathbb{Z}\right\}$. The relation (3.4) then yields

$$
\begin{aligned}
\varphi_{\varepsilon}(z, g)= & (2 \pi)^{(\lambda-\varepsilon) / 2} v^{-\lambda / 2}\left(\chi_{1}(-1)(-1)^{\lambda}+1\right) \sum_{n=1}^{\infty} \chi_{1}(n) n^{\lambda-\varepsilon} v^{\varepsilon} \\
& \times \sum \Theta_{1, \varepsilon}\left(z, g, n((-x) /(8 N)) m, n((-x) /(8 N))\left(-4 N x_{3}^{\prime}\right)\right) \chi_{1}(m) \\
& \times\left(\left(m+4 N x_{3}^{\prime} \bar{z}\right) /(8 \sqrt{N})\right)^{\lambda-\varepsilon} \\
& \times \exp \left(-\pi y^{2} v^{-1} n^{2}\left|\left(m+4 N x_{3}^{\prime} \bar{z}\right) /(8 \sqrt{N})\right|^{2}\right),
\end{aligned}
$$

where the summation is taken over $W_{4 N}$. By using Lemma 3.1, we get

$$
\begin{aligned}
\Theta_{1, \varepsilon}\left(z, g, n((-x)(8 N)) m, n((-x) /(8 N))\left(-4 N x_{3}^{\prime}\right)\right) \\
\quad=\left(\frac{N}{d}\right) J(\sigma, z)^{-1} j(\sigma, z)^{-\varepsilon} \Theta_{1, \varepsilon}(\sigma(z), g,(-x n) /(8 N), 0),
\end{aligned}
$$

where

$$
\sigma=\left(\begin{array}{cc}
a & b \\
4 N x_{3}^{\prime} & m
\end{array}\right) \in \Gamma_{\infty} \backslash \Gamma_{0}(4 N) \quad \text { and } \quad j(\sigma, z)=\left(4 N x_{3}^{\prime} z+m\right) .
$$

Hence, combining those, we see that

$$
\begin{aligned}
\varphi_{\varepsilon}(z, g)= & \left((2 \pi)^{(\lambda-\varepsilon) / 2} v^{-\lambda / 2} v^{\lambda} /(8 \sqrt{N})^{\lambda-\varepsilon}\right)\left(\chi_{1}(-1)(-1)^{\lambda}+1\right) \\
& \times \sum_{n=1}^{\infty} \chi_{1}(n) n^{\lambda-\varepsilon} \sum_{\sigma} \chi_{1}(d)\left(\frac{4 N}{d}\right)\left(J(\sigma, z)^{-1} j(\sigma, z)^{-\lambda}\right) \\
& \times(\operatorname{Im} \sigma(z))^{\varepsilon-\lambda} \Theta_{1, \varepsilon}(\sigma(z), g,(-n x) /(8 N), 0) \\
& \times \exp \left(-\pi y^{2} n^{2}(\operatorname{Im} \sigma(z))^{-1}(8 \sqrt{N})^{-2}\right),
\end{aligned}
$$

where $\sigma=\left(\begin{array}{ll}* & * \\ * & d\end{array}\right)$ and $\sigma$ runs over all elements of $\Gamma_{\infty} \backslash \Gamma_{0}(4 N)$. Consequently, we have the following. 
Lemma 3.3. Suppose that $\bar{\chi}_{1}$ is a primitive character. Then

$$
\begin{aligned}
\Theta(z, g)= & (\sqrt{N} /(2 \sqrt{2 \pi}))^{\lambda} G\left(\bar{\chi}_{1}\right)(1 /(8 \sqrt{N}))\left(1+\chi_{1}(-1)(-1)^{\lambda}\right) \\
& \times \sum_{\varepsilon=0}^{\lambda}\left(\begin{array}{l}
\lambda \\
\varepsilon
\end{array}\right) i^{\lambda}(-1)^{\varepsilon} y^{\lambda-\varepsilon+1} \\
& \times(\sqrt{2 \pi} /(8 \sqrt{N}))^{\lambda-\varepsilon} \sum_{n=1}^{\infty} \chi_{1}(n) n^{\lambda-\varepsilon} \sum_{\sigma}\left(\frac{N}{d}\right) \chi_{1}(\sigma) J(\sigma, z)^{-\kappa} \\
& \times(\operatorname{Im} \sigma(z))^{\varepsilon-\lambda} \Theta_{1, \varepsilon}(\sigma(z), g,(-n x) /(8 N), 0) \\
& \times \exp \left(-\pi y^{2} n^{2}(\operatorname{Im} \sigma(z))^{-1}(8 \sqrt{N})^{-2}\right)
\end{aligned}
$$

where $\sigma=\left(\begin{array}{ll}a & b \\ c & d\end{array}\right), \chi_{1}(\sigma)=\chi_{1}(d)$ and $\sum_{\sigma}$ is the sum taken over all $\sigma \in$ $\Gamma_{\infty} \backslash \Gamma_{0}(4 N)$.

4. Explicit computation of Fourier coefficients of $\Psi(F)$. The purpose of this section is to determine the Fourier coefficients of $\Psi(F)$ in terms of those of $F$ for non-holomorphic modular forms $F$ of half integral weight. Define

$$
\begin{aligned}
W(2 \pi \eta)= & \sum_{\varepsilon=0}^{\lambda} \int_{0}^{\infty}\left(\begin{array}{l}
\lambda \\
\varepsilon
\end{array}\right)(-1)^{\varepsilon}(4 \eta)^{-(\varepsilon-1)}(\sqrt{2 \pi} /(8 \sqrt{N}))^{\lambda-\varepsilon} \\
& \times(1 / \sqrt{2 \pi N})^{\varepsilon-3}(2 \pi N)^{-1} \\
& \times H_{\varepsilon}(2 \sqrt{t}) W(t, \kappa / 2, \nu) \exp (-t) \exp \left(-\pi^{2} \eta^{2}(2 t)^{-1}\right) d t
\end{aligned}
$$

First we investigate the integral

$$
\begin{aligned}
\int_{D} v^{\kappa / 2} & \sum_{\sigma \in \Gamma_{\infty} \backslash \Gamma_{0}(4 N)}\left(\frac{N}{d}\right) \bar{\chi}_{1}(\sigma) J \overline{(\sigma, z)}^{-\kappa}(\operatorname{Im} \sigma(z))^{\varepsilon-\lambda} \\
& \times \bar{\Theta}_{1, \varepsilon}\left(\sigma(z), \sigma_{4 w},(-4 \xi n) /(8 N), 0\right) \\
& \times \exp \left(-\pi(4 \eta)^{2} n^{2}(\operatorname{Im} \sigma(z))^{-1}(8 \sqrt{N})^{-2}\right) F(z) v^{-2} d u d v
\end{aligned}
$$

where $D$ is a fundamental domain for $\Gamma_{0}(4 N), z=u+i v \in \mathfrak{H}, w=\xi+i \eta \in \mathfrak{H}$ and $\sigma_{4 w}=\left(\begin{array}{c}\sqrt{4 \eta} 4 \xi \sqrt{4 \eta}^{-1} \\ 0 \sqrt{4 \eta}^{-1}\end{array}\right) \in \mathrm{SL}(2, \mathbb{R})$. Put $\sigma(z)=z^{\prime}=u^{\prime}+i v^{\prime}$. By changing the order of integration and summation, the above integral is equal to $\sum_{\sigma \in \Gamma_{\infty} \backslash \Gamma_{0}(4 N)} \int_{\sigma(D)} v^{\prime(1 / 2) \kappa+\varepsilon-\lambda} \bar{\Theta}_{1, \varepsilon}\left(z^{\prime}, \sigma_{4 w},(-4 \xi n) /(8 N), 0\right)$ 


$$
\begin{aligned}
& \times \exp \left(-\pi(4 \eta)^{2} n^{2} v^{\prime-1}(8 \sqrt{N})^{-2}\right) F\left(z^{\prime}\right) v^{\prime-2} d u^{\prime} d v^{\prime} \\
= & \int_{0}^{1} \int_{0}^{\infty} v^{\prime(1 / 2) \kappa+\varepsilon-\lambda} \bar{\Theta}_{1, \varepsilon}\left(z, \sigma_{4 w},(-4 \xi n) /(8 N), 0\right) \\
& \times \exp \left(-\pi(4 \eta)^{2} n^{2} v^{-1}(8 \sqrt{N})^{-2}\right) F(z) v^{-2} d u d v .
\end{aligned}
$$

Let $F(z)=\sum_{n \neq 0} a(l) W(2 \pi l v, \kappa / 2, \nu) e[l u] \in S_{\kappa / 2, \nu}\left(4 N, \bar{\chi}\left(\frac{N}{*}\right)\right)$. Then

$$
\begin{aligned}
\int_{0}^{1} \bar{\Theta}_{1, \varepsilon}\left(z, \sigma_{4 w},(-4 \xi n) /(8 N), 0\right) F(z) d u \\
=v^{(-\varepsilon) / 2} \sum_{m=-\infty}^{\infty} H_{\varepsilon}(2 \sqrt{2 \pi N v} m) a\left(N m^{2}\right) \\
\quad \times W\left(2 \pi N m^{2} v, \kappa / 2, \nu\right) \exp \left(-2 \pi N m^{2} v+\pi i(4 \xi m n) / 2\right) .
\end{aligned}
$$

Combining those, we have

$$
\begin{aligned}
\Psi(F)(w)= & c \sum_{m \neq 0} \sum_{n=1}^{\infty} \sum_{\varepsilon=0}^{\lambda} \bar{\chi}_{1}(n) n^{\lambda-1} a\left(N m^{2}\right) \int_{0}^{\infty} n^{-(\varepsilon-1)}\left(\begin{array}{l}
\lambda \\
\varepsilon
\end{array}\right) \\
& \times(-1)^{\varepsilon}(4 \eta)^{-\varepsilon+1}(\sqrt{2 \pi} /(8 \sqrt{N}))^{\lambda-\varepsilon} v^{(\varepsilon-3) / 2} H_{\varepsilon}(2 \sqrt{2 \pi v N} m) \\
& \times W\left(2 \pi N m^{2} v, \kappa / 2, \nu\right) \exp \left(-2 \pi N m^{2} v\right) \\
& \times \exp \left(-(8 \sqrt{N})^{-2} \pi(4 \eta)^{2} n^{2} v^{-1}\right) d v \exp (2 \pi i m n \xi)
\end{aligned}
$$

where

$$
c=(\sqrt{N} /(2 \sqrt{2 \pi}))^{\lambda} G\left(\bar{\chi}_{1}\right)(1 /(8 \sqrt{N}))\left(1+\chi_{1}(-1)(-1)^{\lambda}\right) .
$$

To reduce the above integration, we replace $v$ by $t=2 \pi N m^{2} v$ and conclude

$$
\begin{aligned}
\Psi(F)(w)= & c \sum_{m \neq 0} \sum_{n=1}^{\infty} \bar{\chi}_{1}(n) n^{\lambda-1} a\left(N m^{2}\right) \\
& \times \sum_{\varepsilon=0}^{\lambda} \int_{0}^{\infty}\left(\begin{array}{l}
\lambda \\
\varepsilon
\end{array}\right)(-1)^{\varepsilon}(4 n \eta)^{-(\varepsilon-1)}(\sqrt{2 \pi} /(8 \sqrt{N}))^{\lambda-\varepsilon} \\
& \times(\sqrt{2 \pi N})^{3-\varepsilon}|m|^{-(\varepsilon-3)} \sqrt{t}^{\varepsilon-3}(\operatorname{sgn} m)^{\varepsilon} H_{\varepsilon}(2 \sqrt{t}) W(t, \kappa / 2, \nu) \\
& \times \exp (-t) \exp \left(-\pi 4^{2} \eta^{2} 2 \pi N(m n)^{2} t^{-1}(8 \sqrt{N})^{-2}\right)\left(2 \pi N m^{2}\right)^{-1} d t \\
& \times \exp (2 \pi i m n \xi) .
\end{aligned}
$$

Then we have the following. 
TheOREM 1. Suppose that

$$
F(z)=\sum_{l \neq 0} a(l) W(2 \pi l \eta, \kappa / 2, \nu) e[l \xi] \in S_{\kappa / 2, \nu}\left(4 N, \bar{\chi}\left(\frac{N}{*}\right)\right)
$$

and that $\bar{\chi}_{1}$ is a primitive character and $\kappa \geq 7$. Then

$$
\Psi(F)(w)=c \sum_{l \neq 0} \sum_{n} \bar{\chi}_{1}(n) n^{\lambda-1} \operatorname{sgn}(l / n) a\left(N(l / n)^{2}\right) W(2 \pi l \eta) \exp (2 \pi i l \xi),
$$

where $\sum_{n}$ is the sum taken over all positive divisors $n$ of $l$.

As a consequence of this theorem, we obtain the following corollary.

Corollary. Suppose that $\bar{\chi}_{1}$ is a primitive character and $a(N) \neq 0$. Then there is a constant $c^{\prime}$ such that

$$
\begin{aligned}
\Psi(F)(w)= & c^{\prime} \sum_{l \neq 0} \sum_{n} \bar{\chi}_{1}(n) n^{\lambda-1} \operatorname{sgn}(l / n) a\left(N(l / n)^{2}\right) \\
& \times W(2 \pi l \eta, 2 \lambda, 2 \nu) \exp (2 \pi i l \xi),
\end{aligned}
$$

where $\sum_{n}$ is the sum taken over all positive divisors $n$ of $l$.

Proof. By Proposition 2.1 and Theorem 1, $\Psi(F)$ has the Fourier expansion at infinity

$$
\Psi(F)(w)=\sum_{l \neq 0} b(l) W(2 \pi l \eta, 2 \lambda, 2 \nu) \exp (2 \pi i l \xi) .
$$

Comparing the Fourier coefficients of $\Psi(F)$ and those in Theorem 1, we have

$$
b(1) W(2 \pi \eta, 2 \lambda, 2 \nu)=c a(N) W(2 \pi \eta) .
$$

For $c=0$, our assertion is obvious. Hence we may assume that $c \neq 0$. Then $W(2 \pi \eta)=\beta W(2 \pi \eta, 2 \lambda, 2 \nu)$ for some constant $\beta$, and we obtain the desired result.

An important property of the Shimura correspondence $\Psi$ is that it commutes with the action of Hecke operators. Precisely, we can state this as follows.

THEOREM 2. Under the same notation and assumptions as in Theorem 1 , let $a(N)$ be not zero. If

$$
T_{\kappa / 2, \nu, \bar{\chi}\left(\frac{N}{*}\right)}^{4 N}\left(p^{2}\right) F=\omega_{p} F \quad \text { for a prime number } p,
$$

then

Proof. Put

$$
T_{2 \lambda, 4 \nu, \bar{\chi}^{2}}^{2 N}(p) \Psi(F)=\omega_{p} \Psi(F)
$$

$$
\Psi(F)(w)=\sum_{n \neq 0} b(n) W(2 \pi n \eta, 2 \lambda, 4 \nu) e[n \xi]
$$


and

$$
T_{2 \lambda, 4 \nu, \bar{\chi}^{2}}^{2 N}(p) \Psi(F)(w)=\sum_{n \neq 0} c(n) W(2 \pi n \eta, 2 \lambda, 4 \nu) e[n \xi] .
$$

By Lemma 1.5, we have

$$
c(n)= \begin{cases}b(p n) & \text { if } p \mid n, \\ b(p n)+\bar{\chi}^{2}(p) p^{2 \lambda-1} b(n / p) & \text { otherwise. }\end{cases}
$$

We assume $p \mid n$. We put $n=p^{l} n^{\prime}$ with $\left(p, n^{\prime}\right)=1$. A direct calculation shows that

$$
\begin{aligned}
c(n)= & b(p n)+\bar{\chi}^{2}(p) p^{2 \lambda-1} b(n / p) \\
= & c^{\prime}\left(\sum_{m \mid p n} \bar{\chi}_{1}(m) m^{\lambda-1} \operatorname{sgn}(p n / m) a\left(N(p n / m)^{2}\right)+\bar{\chi}^{2}(p) p^{2 \lambda-1}\right. \\
& \times \sum_{m^{\prime} \mid(n / p)} \bar{\chi}_{1}\left(m^{\prime}\right)\left(m^{\prime}\right)^{\lambda-1} \operatorname{sgn}\left((n / p) / m^{\prime}\right) a\left(N\left((n / p) / m^{\prime}\right)^{2}\right) .
\end{aligned}
$$

By [11, Corollary 1.8],

$$
\begin{aligned}
\sum_{m \mid p n} \bar{\chi}_{1}(m) & m^{\lambda-1} \operatorname{sgn}(p n / m) a\left(N(p n / m)^{2}\right) \\
= & \sum_{\alpha=0}^{l+1} \sum_{m^{\prime} \mid n^{\prime}} \bar{\chi}_{1}\left(p^{\alpha} m^{\prime}\right)\left(p^{\alpha} m^{\prime}\right)^{\lambda-1} \operatorname{sgn}\left(p^{l+1} n^{\prime} / p^{\alpha} m^{\prime}\right) \\
& \times a\left(N p^{2(l+1-\alpha)}\left(n^{\prime} / m^{\prime}\right)^{2}\right) \\
= & \sum_{\alpha=0}^{l-1} \sum_{m^{\prime} \mid n^{\prime}} \bar{\chi}_{1}\left(p^{\alpha} m^{\prime}\right)\left(p^{\alpha} m^{\prime}\right)^{\lambda-1} \operatorname{sgn}\left(p^{l+1} n^{\prime} / p^{\alpha} m^{\prime}\right) \\
& \times\left(\omega_{p} a\left(N\left(p^{l} n^{\prime} / p^{\alpha} m^{\prime}\right)^{2}\right)-\bar{\chi}^{2}(p) p^{\kappa-2} a\left(N\left(p^{l-1} n^{\prime} / p^{\alpha} m^{\prime}\right)^{2}\right)\right) \\
& +\sum_{m^{\prime} \mid n^{\prime}} \bar{\chi}_{1}\left(p^{l} m^{\prime}\right)\left(p^{l} m^{\prime}\right)^{\lambda-1} \operatorname{sgn}\left(p n^{\prime} / m^{\prime}\right) a\left(N p^{2}\left(n^{\prime} / m^{\prime}\right)^{2}\right) \\
& +\sum_{m^{\prime} \mid n^{\prime}} \bar{\chi}_{1}\left(p^{l+1} m^{\prime}\right)\left(p^{l+1} m^{\prime}\right)^{\lambda-1} \operatorname{sgn}\left(n^{\prime} / m^{\prime}\right) a\left(N\left(n^{\prime} / m^{\prime}\right)^{2}\right) .
\end{aligned}
$$

Now we have

$\sum_{m^{\prime} \mid n^{\prime}} \bar{\chi}_{1}\left(p^{l} m^{\prime}\right)\left(p^{l} m^{\prime}\right)^{\lambda-1} \operatorname{sgn}\left(p n^{\prime} / m^{\prime}\right) a\left(N p^{2}\left(n^{\prime} / m^{\prime}\right)^{2}\right)$ 


$$
\begin{aligned}
= & \bar{\chi}_{1}\left(p^{l}\right)\left(p^{l}\right)^{\lambda-1} \sum_{m^{\prime} \mid n^{\prime}} \bar{\chi}_{1}\left(m^{\prime}\right)\left(m^{\prime}\right)^{\lambda-1} \operatorname{sgn}\left(n^{\prime} / m^{\prime}\right) \\
& \times\left(\omega_{p} a\left(N\left(n^{\prime} / m^{\prime}\right)^{2}\right)-\left(\frac{\left(n^{\prime} / m^{\prime}\right)^{2} N}{p}\right) \bar{\chi}_{1}(p)\left(\frac{N}{p}\right) p^{\lambda-1} a\left(N\left(n^{\prime} / m^{\prime}\right)^{2}\right)\right) .
\end{aligned}
$$

Consequently, we conclude that

$$
c(n)=\omega_{p} b(n) \quad \text { for every } n .
$$

Since we can also verify the remainders, we may omit the details. The proof is complete.

Here we show an example of a non-holomorphic Maass wave cusp form satisfying the conditions of the Corollary to Theorem 1 and of Theorem 2. We denote by $S_{(2 k+1) / 2}(4 N)$ the space of holomorphic modular cusp forms of weight $(2 k+1) / 2$ with respect to $\Gamma_{0}(4 N)$. Put

$$
f(z)=\theta(z)^{-3} \eta(2 z)^{12} \quad \text { with } \eta(z)=e[z / 24] \prod_{n=1}^{\infty}(1-e[n z]) .
$$

Then $f(z)$ belongs to $S_{9 / 2}(4)$ and $f(z)$ generates $S_{9 / 2}(4)$ (cf. Shimura [11, p. 477]). Moreover, $\theta(z)^{2} f(z)$ is an element of $S_{11 / 2}(4)$ and $\theta(z)^{2} f(z)$ generates $S_{11 / 2}(4)$, where $\theta(z)=\sum_{n=-\infty}^{\infty} e\left[n^{2} z\right]$. We may check that $\widetilde{f}(z)=$ $\delta_{11 / 2}\left(\theta(z)^{2} f(z)\right)$ belongs to $S_{15 / 2,-11 / 2}\left(4, \chi_{0}\right)$ and $\widetilde{f}(z)$ is an eigenfunction of Hecke operators of $S_{15 / 2,-11 / 2}\left(4, \chi_{0}\right)$ (cf. Friedberg [4, Proposition 1.3]). Therefore $\widetilde{f}(z)$ gives us an example satisfying the conditions of the Corollary to Theorem 1 and of Theorem 2, where $\chi_{0}$ is the trivial character modulo 4 .

5. Remark on cuspidal properties of $\Psi$. In this section we prove the following theorem.

TheOREM 3. Suppose that a function

$$
F=\sum_{n \neq 0} a(n) W(2 \pi n \eta, \kappa / 2, \nu) e[n \xi]
$$

in $S_{\kappa / 2, \nu}(4, \bar{\chi})$ satisfies $a(1) \neq 0$. Then $\Psi(F) \in S_{2 \lambda, 4 \nu}\left(2, \chi^{2}\right)$.

Pr o o f. We can take 0 and $\infty$ to be a complete set of inequivalent classes of cusps of $\Gamma_{0}(2)$. By the Corollary, for some $\delta>0$,

$$
\Psi(F)(w)=O\left(e^{-\delta \operatorname{Im} w}\right)
$$

uniformly in $\operatorname{Re} w$ as $\operatorname{Im} w \rightarrow 0$. To verify our assertion, it is enough to check the behavior of $\Psi(F)$ at 0 . Put $\Phi(w)=\Psi(F)((-1) /(2 w)) 2^{\lambda}(2 w)^{-2 \lambda}$. Then, by the same method as in the case of holomorphic modular forms, we can show that $\Phi(w) \in G_{2 \lambda, 4 \nu}\left(2, \chi^{2}\right)$. By Lemma 1.1, $\Phi(w)$ has the Fourier 
expansion at infinity

$$
\Phi(w)=a_{0}(\eta)+\sum_{n \neq 0} a(n) W(2 \pi n \eta, 2 \lambda, 2 \nu) e[n \xi]
$$

for every $w=\xi+i \eta \in \mathfrak{H}$. By the same method as in [7], we have $|\Psi(F)(w)| \leq M \eta^{-\lambda}\left(\eta+\eta^{-1}\right) \quad$ for every $w \in \mathfrak{H}$ and for some constant $M$.

Therefore

$$
|\Phi(w)| \leq M\left(2^{-\lambda}\right)^{2} \eta^{-\lambda}\left(\eta\left(2|w|^{2}\right)^{-1}+2|w|^{2} \eta^{-1}\right) .
$$

Observe that $\eta\left(2|w|^{2}\right)^{-1} \leq 1 /(2 \eta)$ and $2|w|^{2} \eta^{-1} \leq 2 \eta^{-1}\left(1+\eta^{2}\right)$ for every $\xi(|\xi| \leq 1 / 2)$. Combining these, we have

$$
|\Phi(w)| \leq M\left(2^{-\lambda}\right)^{2} \eta^{-\lambda}\left((2 \eta)^{-1}+2 \eta^{-1}+2 \eta\right) \quad \text { for every } \xi(|\xi| \leq 1 / 2) .
$$

Since $\Phi(w+1)=\Phi(w)$, we have, for some $\alpha>0$ and $M^{\prime}>0$,

$$
|\Phi(w)| \leq M^{\prime} \eta^{-\alpha} \quad \text { uniformly in } \xi \text { as } \eta \rightarrow 0 .
$$

Therefore, by Lemma 1.3, there is a constant $\beta$ with $|a(n)|=O\left(|n|^{\beta}\right)$. This leads to

$$
\left|\sum_{n \neq 0} a(n) W(2 \pi n \eta, 2 \lambda, 4 \nu) e[n \xi]\right|=O\left(e^{-c \eta}\right) \quad \text { as } \eta \rightarrow \infty \text { for some } 0<c \in \mathbb{R} \text {. }
$$

On the other hand, by [7, p. 158 and p. 159], we obtain

$$
\Phi(i \eta)=O\left(\eta^{-\mu}\right) \quad \text { for every } \eta>1 \text { and every } \mu>0 .
$$

From the results above, we have

$$
a_{0}(\eta)=O\left(\eta^{-\mu}\right) \quad \text { for every } \eta>1 \text { and every } \mu>0 .
$$

We can put $a_{0}(\eta)=c_{1} \eta^{((1-2 \lambda) / 2+\omega)}+c_{2} u(\eta, 2 \lambda, 4 \nu)$, where $c_{1}$ and $c_{2}$ are constants and $\omega=\left((2 \lambda-1)^{2}+16 \nu\right)^{1 / 2} / 2$. By the above result, we have $c_{1}=c_{2}=0$. Consequently, $a_{0}(\eta)=0$. This completes the proof.

\section{References}

[1] T. Asai, On the Doi-Naganuma lifting associated with imaginary quadratic fields, Nagoya Math. J. 71 (1978), 149-167.

[2] K. Doi and T. Miyake, Automorphic Forms and Number Theory, Kinokuniya Book Store, 1974 (in Japanese).

[3] S. Friedberg, On the imaginary quadratic Doi-Naganuma lifting of modular forms of arbitrary level, Nagoya Math. J. 92 (1983), 1-20.

[4] -, On Maass wave forms and the imaginary quadratic Doi-Naganuma lifting, Math. Ann. 263 (1983), 483-508.

[5] S. Gelbert and I. Piatetski-Shapiro, On Shimura's correspondence for modular forms of half-integral weight, in: Proc. Internat. Colloq. Automorphic Forms, Representation Theory and Arithmetic, 1979, Tata Institute, Springer, 1981, 1-39. 
[6] D. A. Hejhal, Some Dirichlet series with coefficients related to periods of automorphic eigenforms, Proc. Japan Acad. 58 (1982), 413-417.

[7] S. Niwa, Modular forms of half integral weight and the integral of certain thetafunctions, Nagoya Math. J. 56 (1974), 147-161.

[8] T. Oda, On modular forms associated with indefinite quadratic forms of signature $(2, n-2)$, Math. Ann. 231 (1978), 97-144.

[9] S. Rallis and G. Schiffmann, On a relation between $\widetilde{\mathrm{SL}}$ cusp forms and cusp forms on tube domains associated to orthogonal groups, Trans. Amer. Math. Soc. 263 (1981), 1-58.

[10] P. Sarnak, Additive number theory and Maass forms, Lecture Notes in Math. 1052, Springer, Berlin, 1984, 286-309.

[11] G. Shimura, On modular forms of half integral weight, Ann. of Math. 97 (1973), 440-481.

[12] T. Shintani, On construction of holomorphic cusp forms of half integral weight, Nagoya Math. J. 58 (1975), 83-126.

[13] J.-L. Waldspurger, Correspondence de Shimura, J. Math. Pures Appl. 59 (1980), $1-133$.

DEPARTMENT OF MATHEMATICS

THE UNIVERSITY OF ELECTRO-COMMUNICATIONS

CHOFU-SHI

TOKYO 182, JAPAN 